\title{
Percepción de Blended Learning en profesores universitarios de distintos ámbitos disciplinares
}

\author{
António Martín García, María Cruz Sánchez \\ Gómez \& António Pedro Costa
}

Resumen:

El papel de los profesores en el proceso de mejorar la eficacia y eficiencia de la integración de nuevos enfoques pedagógicos y el potencial que ofrecen los recursos tecnológicos es básico para introducir un cambio sustancial en los procesos de enseñanza-aprendizaje, en el contexto de la educación superior. En esta línea, el objetivo de este estudio es conocer las experiencias y percepciones de profesores universitarios de distintos ámbitos disciplinares (ramas de conocimiento) sobre aceptación y adopción de metodologías basadas en Blended Learning (BL). El trabajo empírico se realizó siguiendo un diseño metodológico mixto de tipo derivativo secuencial de equivalencia de estatus. La información cuantitativa fue recogida con un cuestionario, elaborado ad hoc, basado en el marco teórico propuesto por los Modelos de Aceptación Tecnológica (TAM), al que contestaron 980 profesores universitarios; de éstos, 86 manifestaron de forma abierta su opinión sobre estas metodologías, constituyendo la parte cualitativa objeto de este estudio. Los resultados ponen de manifiesto a juicio de los docentes participantes en el estudio, las debilidades, amenazas, fortalezas y oportunidades de la enseñanza B-learning.

Palabras clave:

blended learning; innovación tecnológica; educación superior; metodología cualitativa, caqdas. 


\title{
Perception of blended learning by university teachers of different disciplinary areas
}

\begin{abstract}
The role of teachers in the process of improving the effectiveness and efficiency of the integration of new pedagogical approaches and the potential offered by technological resources is essential to introduce a substantial change in teaching-learning processes, in higher education. Based on this idea, the objective of this study is to learn the experiences and perceptions of university teachers from different disciplinary areas (knowledge branches) about the acceptance and adoption of Blended Learning $(\mathrm{BL})$ methodologies. The empirical work was carried out following a mixed methodological design of the sequential derivative type of equivalence of status. The quantitative information was collected with a questionnaire, elaborated ad hoc, based on the theoretical framework proposed by the Technological Acceptance Models (TAM), answered by 980 university teachers; of these, 86 expressed their opinion openly on these methodologies, constituting the qualitative part of this study. The results show the opinion of the teachers participating in the study regarding the weaknesses, threats, strengths and opportunities of B-learning education.
\end{abstract}

Keywords: blended learning; technological innovation; higher education; qualitative methodology; caqdas.

\section{Percepção do Blended Learning de professores universitários de diferentes áreas disciplinares}

Resumo: O papel dos professores no processo de melhoria da eficácia e eficiência da integração de novas abordagens pedagógicas e do potencial oferecido pelos recursos tecnológicos é essencial para introduzir uma mudança substancial nos processos de ensino-aprendizagem, no ensino superior. A partir desta ideia, o objetivo deste estudo é conhecer as experiências e percepções de professores universitários de diferentes áreas disciplinares (ramos do conhecimento) sobre a aceitação e adoção de metodologias baseadas em Blended Learning (BL). O trabalho empírico foi realizado seguindo um desenho metodológico misto do tipo derivativo sequencial de equivalência de status. As informações quantitativas foram coletadas com um questionário, elaborado ad hoc, com base no referencial teórico proposto pelos Modelos de Aceitação Tecnológica (TAM), respondido por 980 professores universitários; destes, 86 expressaram abertamente a sua opinião sobre estas metodologias, constituindo a parte qualitativa deste estudo. Os resultados mostram, a opinião dos professores participantes do estudo a respeito das fragilidades, ameaças, pontos fortes e oportunidades da educação B-learning.

Palavras Chave: blended learning; inovação tecnológica; ensino superior; metodologia qualitativa; caqdas.

\section{La perception de Blended Learning chez les professeurs d'université de différents domaines disciplinaires}

Résumé: Afin de promouvoir un changement significatif du processus d'enseignement-apprentissage dans l'éducation supérieure, le rôle des enseignants est crucial; c'est à eux que revient l'intégration des nouvelles approches pédagogiques et du potentiel des ressources technologiques, ainsi que l'amélioration de leur efficacité et de leur efficience. L'objectif de ce travail est de connaître les expériences et les perceptions des enseignants universitaires de différents domaines de la connaissance à propos de l'acceptation et de l'adoption des méthodologies basées sur le Blended Learning (BL). Le travail empirique a été effectué selon une méthodologie mixte d'équivalence de statut suivant une approche " séquentielle-dérivative ". L'information quantitative a été obtenue au moyen d'un questionnaire élaboré ad hoc, basé sur le cadre théorique proposé par les Modèles d'Acceptation Technologique (TAM), auquel ont répondu 980 enseignants universitaires, dont 86 ont exprimé ouvertement leur opinion sur ces méthodologies ; leurs avis constituent la partie qualitative qui fait l'objet de cet article. Les résultats mettent en évidence la perception des enseignants participant à cette étude à l'égard des faiblesses, des menaces, des forces et des enjeux de l'enseignement du B- learning.

Mots-clés: apprentissage mixte; innovation technologique; enseignement supérieur; méthodologie qualitative; caqdas. 


\section{Introducción}

Existe un amplio acuerdo internacional en señalar que la formación basada en metodologías blended learning (B-Learning o BL) presenta características que la convierten en una excelente oportunidad para introducir un cambio paradigmático en la educación superior, siendo destacado en los informes internacionales de los últimos años como el modelo de educación del siglo XXI (Garrison \& Vaughan,2007; Becker, Cummins, Davis, Freeman, Hall, \& Ananthanarayanan, 2017). Por este motivo, los enfoques que combinan instrucción basada en comunicación cara a cara con métodos online están aumentando en las universidades de todo el mundo, gracias también a la mejora de las plataformas de gestión del aprendizaje (Learning management system, LMS; LCMSC, Learning Content Management), así como al avance en el desarrollo de nuevas innovaciones docentes, que permiten combinar de manera efectiva determinados recursos tecnológicos con renovados enfoques pedagógicos. Ejemplo de ello son las prácticas educativas basadas en el aprendizaje invertido (Flipped Learning, a partir de técnicas como el Just in Time Teaching (JITT) (Peer Instruction (P-I) gamificación, la creación de micromundos, el aprendizaje situado, las comunidades de aprendizaje, aprendizaje por simulación, e-mentoring, etc. que se suman a otras metodologías más consolidadas basadas en el Aprendizaje colaborativo asistido por computadora (CSCL, Computer Supported Collabortive Learning), el aprendizaje basado en proyectos, el aprendizaje centrado en escenarios, los entornos constructivistas de aprendizaje, etc. El desarrollo y expansión de todo este conjunto de nuevos sistemas de formación marca un claro proceso de virtualización en el que está sumida ya la enseñanza superior en todo el mundo y aumenta la necesidad de promover estudios que analicen diferentes aspectos relacionados con la adopción y aceptación de la metodología BL a partir de la integración o combinación de las modalidades virtual y presencial.

El análisis sobre el proceso de adopción de las innovaciones educativas ha sido realizado desde diferentes perspectivas, pero se asume que en general se produce mediante una secuencia de aceptación de las mismas que se da de manera gradual y por etapas. Según esto, los profesores pasan de un estado de mínimo contacto con la innovación a uno final en el que el propósito es la integración normalizada de ésta en la práctica cotidiana docente. En la literatura se pueden encontrar varios enfoques que consideran la innovación como un proceso individual de cambio por etapas (Sánchez, 2003; Rogers, 2003; Hall, 2014; etc.), donde, como decimos, hay un momento inicial en el que el docente conoce o toma conciencia de la existencia de la innovación y, después de una o más etapas, se involucra de manera efectiva en ella, construyendo nuevas prácticas a través de la reflexión y la experimentación, llegando finalmente a una etapa en la que es capaz de integrar eficazmente la innovación y aplicarla a diferentes procesos de formación. 
Ese proceso implica una secuencia que el docente recorre a partir de decisiones de tipo individual. Pero otros enfoques han analizado la adopción de innovaciones didácticas y/o tecnológicas también desde la perspectiva de las organizaciones. Por ejemplo, el meta-modelo clásico planteado por Saga y Zmud (1994) ofrece un panorama sistémico para comprender los elementos que intervienen, desde la aceptación tecnológica hasta la infusión (apropiación) de las tecnologías. Esta perspectiva institucional describe tres etapas principales: Aceptación voluntaria (en esta etapa, lo importante son las creencias, las actitudes y la intención del personal académico sobre utilizar la innovación); Rutinización (la tecnología se considera un elemento estándar de la rutina normal de una organización. Es decir, en esta fase la innovación es institucionalizada); y la Infusión o Apropiación (se trata del momento de integración profunda y completa de una aplicación tecnológica en los sistemas de trabajo de una organización, que afecta a elementos como son la frecuencia de uso, el uso estandarizado, intervención administrativa, reingeniería de procesos, comunicación y conocimiento del usuario, etc.). Para Saga y Zmud, este es el momento a partir del cual la organización comienza a reconceptualizar ciertos procesos a través de la innovación que se ha incorporado.

En el contexto concreto de la universidad pública española parece evidente que gran parte de este proceso de integración de la innovación BL (nos referiremos aquí como sistemas o entornos BL) depende de la decisión individual y voluntaria de los profesores, decisión que puede verse mediatizada por factores "corporativos" o, más propiamente, disciplinares. Es decir, la secuencia de fases o etapas por las que transita la adopción de una innovación docente puede estar mediatizada no solo por una serie de características individuales del profesorado (que han sido ampliamente analizadas en la investigación sobre el tema, como el sexo, la edad, experiencia docente, etc.), sino también por rasgos grupales, relacionadas con un perfil académico determinado. En la universidad estos rasgos aparecen reunidos en Ramas de Conocimiento que definen determinados grupos de disciplinas científicas. Muchas de ellas coinciden en los modos de hacer investigación, pero también, asumimos aquí, de ejercer la práctica docente. Precisamente, esta hipótesis es la que tratamos de analizar en nuestro trabajo a propósito de la aceptación de una innovación docente como es la metodología basada en blended learning.

\section{Método}

\subsection{Objetivos y pregunta de investigación}

A partir del planteamiento general expresado anteriormente nos proponemos como objetivo principal de este estudio desarrollar un proceso de análisis mixto (CUANCUAL) entorno a la siguiente pregunta de investigación: ¿existen diferencias entre los 
docentes universitarios sobre la percepción de uso de la metodología blended learning en función del ámbito disciplinar o rama de conocimiento a la que pertenecen?

\subsection{Diseño y método}

Para dar respuesta a esta cuestión y conseguir el objetivo propuesto se ha seguido un diseño de carácter mixto "secuencial de equivalencia de estatus", que combina los métodos cuantitativo y cualitativo, y guían la construcción y el desarrollo del estudio empírico. La comparación obliga a establecer diferenciaciones no sólo en términos de cantidad (cuantitativas), sino también de cualidad (cualitativas) (Hernández Sampieri et al, 2014; Creswell, 2013; Sánchez-Gómez y Martín-García, 2017). En concreto, se utilizó el modelo explicativo secuencial DEXPLIS (Creswell, 2013) donde en una primera fase se recolectaron y analizaron datos cuantitativos, y en la segunda se recogieron y evaluaron los cualitativos. El nexo o conexión entre ambas metodologías ocurre cuando los resultados cuantitativos iniciales sirven para recoger datos cualitativos. La segunda fase se construye sobre los resultados de la primera y ambos se integran en las conclusiones. De esta forma, se clarifican los resultados de un método sobre la base de los del otro, abordándose el problema de forma completa e integral. (Anguera, Blanco-Villaseñor, Losada; Sánchez-Algarra; \& Onwuegbuzie, 2018; Levitt, Bamberg; Creswell, Frost; Josselson, \& Suárez-Orozco, 2018). En el diseño cuantitativo se utilizó el método no experimental o ex post facto y en el cualitativo se recurrió a la teoría fenomenológica para construir el sentir de los participantes desde su formación, experiencia y sobre los significados dados a su docencia universitaria. Con este fin, las respuestas fueron analizadas mediante la técnica de análisis de contenido clásico con ayuda del programa NVIVO12. En este artículo únicamente se presentarán los resultados resultantes del estudio cualitativo.

\subsection{Estudio cualitativo}

- Participantes, técnicas de recogida de información y análisis

Se aplicó mediante envío on-line un cuestionario a 980 profesores de universidades públicas de todo el territorio español (481 profesores y 499 profesoras), el 71,8 \% tiene más de 10 años de experiencia docente en la universidad. Por Rama de Conocimiento, la muestra se dividió en Ciencias Sociales y Jurídicas (390 docentes); Ciencias de la Salud ( $n=154)$, Arquitectura e Ingeniería $(n=132)$, Ciencias $(n=150)$ y Arte y Humanidades $(n=156)$. Por último, el $41,0 \%$ de la muestra afirma haber recibido cursos de formación en B-Learning, mientras que el 48,7\% indica explícitamente no haber recibido formación específica en esta materia. Una vez aplicado el cuestionario, se procedió a realizar los análisis estadísticos pertinentes, con el fin de tener una visión cuantitativa y generalizable de las cuestiones planteadas. Para profundizar en algunos tópicos y obtener significados relevantes sobre las respuestas obtenidas en las escalas likert, se 
elaboraron preguntas abiertas, y se les invitó a que informaran o añadieran desde su experiencia, alguna reflexión sobre la enseñanza B_learning, cometando además, las dificultades, ventajas y/o sugerencias de mejora hacia esta metodología. Teniendo en cuenta las consideraciones anteriores respondieron 86 docentes universitarios. Estas técnicas de recogida de información permitieron, entre otras cuestiones, determinar el uso innovador del B_learning y los cambios producidos en los procesos de enseñanza aprendizaje, valorar la aceptación de su uso, indicar si los docentes han sido capaces de adaptarse con rapidez a este nuevo método en el entorno virtual universitario, informar sobre la necesidad de formación previa, actitudes y expectativas, ventajas, dificultades y demandas hacia este tipo de enseñanza.

La interpretación de los comentarios del profesorado se efectuó conforme al proceso analítico básico o común a la mayoría de las investigaciones cualitativas basado en el esquema general de Miles y Huberman (1994): a) reducción de datos; b) disposición y transformación de datos; y c) obtención de resultados y verificación de conclusiones, Además, para describir de forma exhaustiva la información recogida se ha realizado previamente un análisis de frecuencia de palabras.
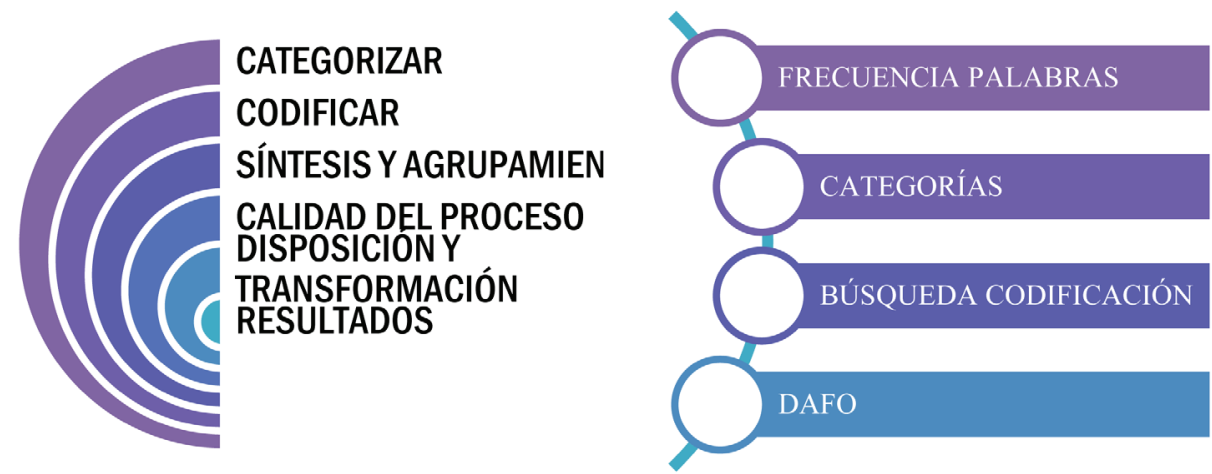

Figura 1. Esquema analítico seguido en el estudio cualitativo

En el análisis de contenido de los textos producidos en los correos electrónicos se obtuvieron resultados que hacen referencia al porcentaje e interpretación de los comentarios analizados, que corresponden a cada una de las categorías propuestas en el mapa conceptual. En la primera fase del análisis cualitativo (categorización) se identificaron las ideas/categorías más importantes manifestadas por los docentes universitarios. Como resultado, se elaboró el mapa conceptual del que presentamos las categorías principales. El análisis de esta información, permitió reducirla y organizarla en un mapa conceptual constituido por unidades de información, categorías, 
dimensiones y conceptos integradores que logran generar teoría a partir de la reflexión sobre y desde la práctica (figura 2).

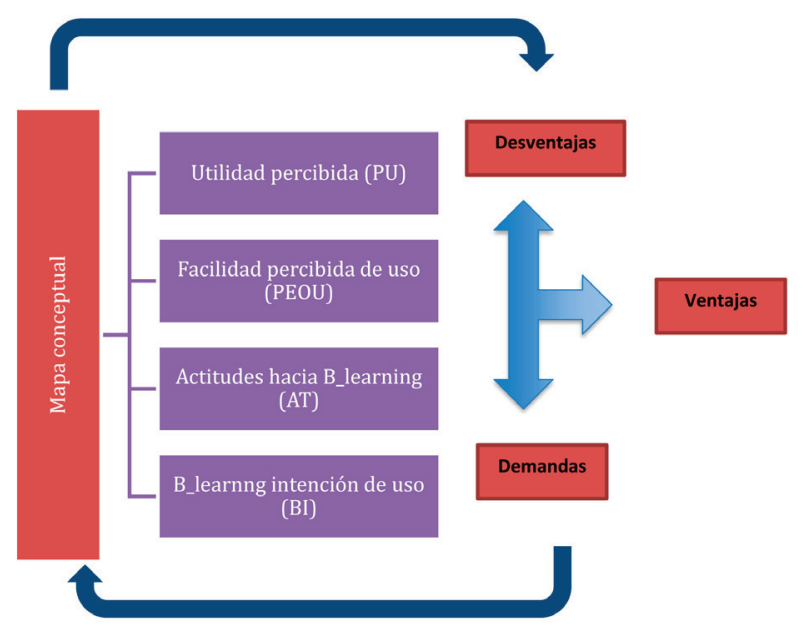

Figura 2. Mapa conceptual de las opiniones del profesorado sobre la enseñanza B_learning

En la segunda fase del análisis se realizó la codificación axial y selectiva. Posteriormente y para la organización, clasificación y disposición de los datos textuales codificados en tablas y gráficas, se utilizó el programa análisis cualitativo NVIVO12. Durante esta fase se ha garantizado en todo momento los criterios de calidad en investigación cualitativa (Guba y Lincoln, 1989; Sánchez Gómez y Martin García, 2017) que quedan sintetizados como sigue: la codificación se realizó por varios miembros del equipo de investigación para así poder garantizar la credibilidad (validez interna) del proceso. Para probar la dependencia (fiabilidad) del sistema de categorías se elaboró un manual descriptivo de las mismas para realizar la codificación. Por último, la objetividad (Confirmabilidad) se ve reflejada en los resultados mediante la aportación de fragmentos literales del discurso de los docentes.

Recogiendo las definiciones conceptuales de los indicadores principales que forman parte de los modelos TAM (Utilidad Percibida, Facilidad Percibida de Uso, Condiciones Facilitadoras, Actitud hacia b-learning, etc.) tomados de la literatura de referencia (TAM, Technology Acceptance Model), se identificaron las categorías en los textos (proceso de codificación). 
Y finalmente, en la tercera fase del análisis, se describen los resultados de los análisis de contenido de las opiniones sobre la enseñanza B_learning de los docentes universitarios que contestaron a los mails realizando fruto de un procedimiento inductivo y reflexivo.

\subsection{Resultados}

\section{- Análisis cualitativo}

Del total de la muestra de profesores se seleccionaron aquellos que cumplimentaron el apartado de preguntas abiertas obteniéndose un total de 86 respuestas, que sirven de base para el análisis cualitativo. La temática de estas preguntas gira en torno a ventajas, desventajas y demandas de la metodología BL. El esquema de presentación de los datos se efectuará siguiendo los siguientes parámetros: análisis semántico del léxico (significado/frecuencia ocurrencia) y análisis de contenido cualitativo.

\section{-Resultados del análisis semántico}

Si analizamos la frecuencia de ocurrencia de las palabras considerando las dimensiones ventajas, desventajas y demandas de la metodología de enseñanza B_learning, se aprecian en los comentarios de los docentes universitarios estas diferencias:
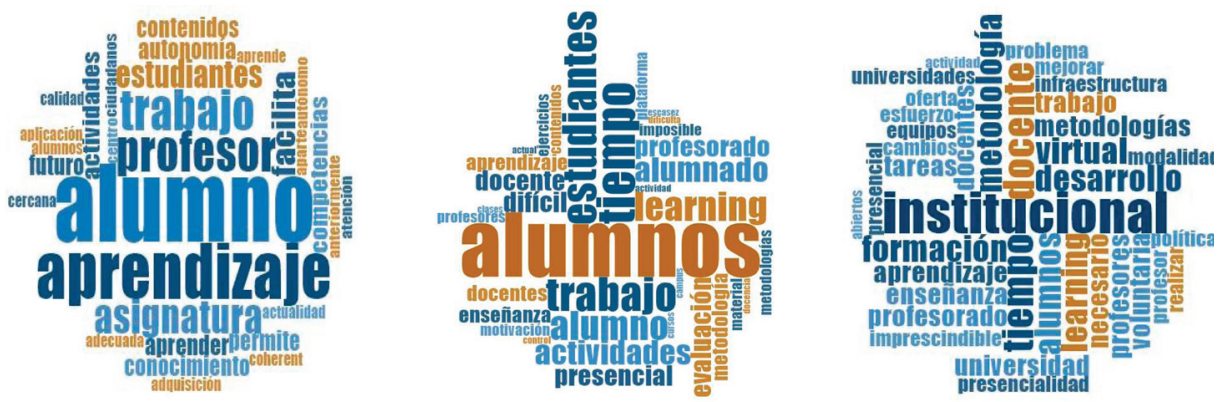

Figura 3. Frecuencia de ocurrencia de palabras

En la "nube de palabras" (figura 3) se comprueba que tanto en las ventajas como las desventajas la alusión a los estudiantes es mayoritaria, mientras que en las demandas las reivindicaciones se centran en la institución universitaria.

La frecuencia de ocurrencia de palabras significativas en las ventajas del uso de la enseñanza B_learning son principalmente alumno, profesor y trabajo. En cuanto al "alumno" piensan que le acerca más a la asignatura, se tiene en cuenta su ritmo de aprendizaje, tiene mayor feedback con el-profesor, más autonomía, motivación y responsabilidad en el proceso educativo, y favorece el trabajo a distancia. En cuanto al "profesor", refuerzan la idea de la mayor interacción, el trabajo a distancia y obliga a los docentes a estructurar y presentar el contenido de las asignaturas de forma práctica 
y coherente. Por lo que respecta al "trabajo", piensan que favorece el cooperativo y el aprendizaje significativo. Las palabras más frecuentes cuando hablan de desventajas son alumno/s/estudiante/s, tiempo y trabajo. De los primeros dicen que es difícil contabilizar sus horas de trabajo y evaluarles, además existe el problema de la identificación de quien realiza las actividades por el tema de suplantación de identidad, piensan que se hacen más vagos, pierden interés y se desmotivan con mayor facilidad. Al contrario que algunos docentes que señalan como ventaja la mayor interacción entre el profesor y el alumno, algunos piensan que no es así y que es menor el flujo de comunicación entre ambos. Además, piensan que con la ratio actual que hay en las aulas universitarias españolas es muy difícil implementar este tipo de enseñanza. En cuanto al "tiempo" piensan que la preparación de estas clases es excesiva, no está incentivado y supone un esfuerzo muy costoso.

Por lo que respecta a las "demandas", éstas se centran fundamentalmente en la institución universitaria española, la necesidad de apoyo y formación. La mayor parte piensan que no hay financiación ni acompañamiento al docente para su puesta en práctica. Creen que debe haber una estrategia institucional formal que apoye en infraestructuras tecnológicas, legislación, subvención de proyectos innovadores, y planes de formación docentes específicos. Consideran que en la actualidad ésta es escasa y poco práctica.

\section{- Búsqueda de codificación}

Una vez realizado el análisis de contenido el total de referencias de codificación en las tres dimensiones analizadas los resultados ponen de manifiesto que los docentes participantes en el estudio encuentran más inconvenientes que ventajas a la hora de utilizar la metodología b-learning en la enseñanza universitaria:

\section{A : Ventajas}

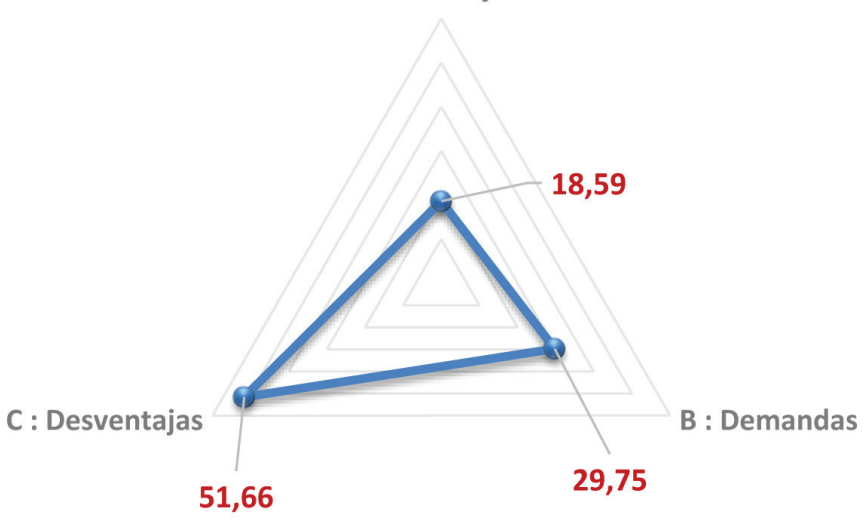

Figura. 4. Frecuencia de ocurrencia categorías 
Si seleccionamos los comentarios de los docentes en función de las ramas de conocimiento se percibe que el mayor número de comentarios que se refieren a las dificultades de la utilización de la enseñanza $B \_L$ se encuentran en los docentes de las titulaciones de Ciencias, seguidos de las Ingenierías y Arquitectura; los que menos inconvenientes relatan son los de Arte y Humanidades. Por su parte, Ciencias de la Salud y CCSS y Jurídicas son los que comentan más factores positivos y los de CCSS y Jurídicas los que exponen más demandas.

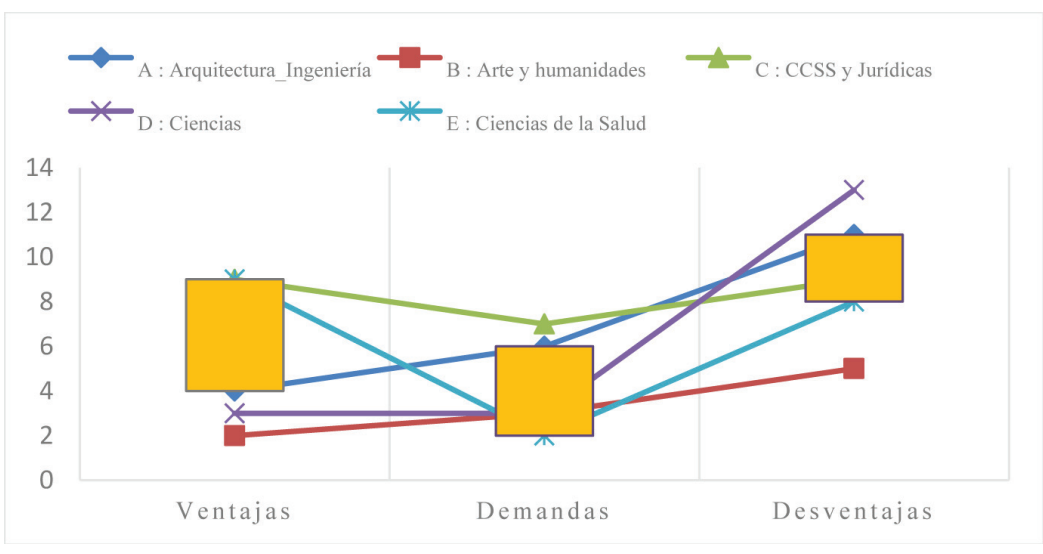

Fig. 5. Análisis de contenido

(Referencias de codificación por dimensiones_ramas de conocimiento)

Al transponer los datos observamos que menos en CCSS, Jurícicas y de la Salud, los demás docentes encuentran más dificultades que ventajas a la hora de implementar el tipo de enseñanza B_Learnig.

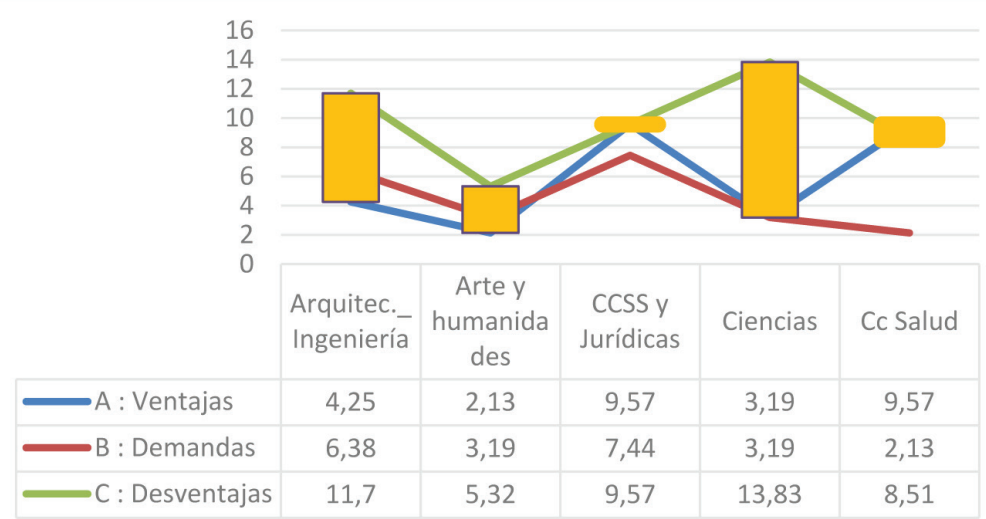

Fig. 6. Análisis de contenido

(\% Referencias de codificación por ramas de conocimiento_dimensiones) 
Los factores positivos señalados por los docentes de Ciencias Sociales, Jurídicas y de la Salud son el acercamiento a la realidad social, facilidad en casos prácticos y gestión de la documentación, fortalecimiento de competencias digitales, autonomía personal y trabajo cooperativo, mayor interacción profesor -alumno y aprendizaje significativo. Además, potencia la comunicación y mejora los procesos de evaluación. "Es indispensable en la actualidad en la formación superior "(docente de Ciencias Sociales y Jurídicas) "Considero que es imprescindible para desarrollar competencias digitales, de aprender a aprender, autonomía personal, para el trabajo cooperativo, etc., "(docente en Ciencias Sociales y Jurídicas). "Es la mejor forma de evaluación formativa y de utilizar los recursos que los estudiantes comparten" (docente en Ciencias de la Salud). "El uso de la metodología virtual en la universidad sitúa a esta institución hace que esta evolucione en paralelo a la sociedad a la cual debe servir, y no se queda retrasada, ya que estas metodologías telemáticas y redes sociales en internet son de uso común en la vida cotidiana de nuestros ciudadanos" (docente en Ciencias de la Salud).

Las desventajas que subrayan los docentes de las titulaciones de Ciencias se refieren sobre todo en temas relacionados con la ratio tan elevada de estudiantes por grupo, más tiempo y esfuerzo personal, importancia del contacto presencial frente al virtual, pésima coordinación entre docentes, suplantación de personalidad entre estudiantes, dificultad para contabilizar las horas en las actividades que desarrolla el estudiante de forma no presencial, dificultad de integral estas actividades en el proceso de evaluación y la escasa motivación y el alto porcentaje de abandono de los estudiantes. "te da más trabajo y nadie lo reconoce. Los que menos, los estudiantes "(docente en Ciencias). "Un altísimo porcentaje de mis alumnos asisten a academias o pasantías para que les ayuden a superar la materia. Es un mal endémico. No hay ninguna garantía de que cualquier tarea que se realice y/o entregue fuera del horario de clases sea hecha realmente por ellos. De ahí que haya abandonado la idea del BL como apoyo para la evaluación continua hace muchísimo tiempo. He probado en los últimos años a ponerles algún cuestionario de autoevaluación (por supuesto, sin que su resolución tenga ninguna repercusión en la nota final) y el resultado ha sido desolador (no han entrado en él más que 2-3 alumnos cada año)" (docente en Ciencias)

Por lo que se refiere a las demandas, los profesores de ingeniería y arquitectura reclaman política institucional, correctamente diseñada y financiada, que esté valorada en su justa medida, apoyo en infraestructuras y formación. Los de Ciencias Sociales y Jurídicas reclaman, además, normativa específica e iniciativas de formación docente desde las facultades "El uso de estas metodologías no puede dejarse a voluntad de cada profesor. Debe ser una política institucional, correctamente diseñada y financiada, con un plan de desarrollo correctamente ideado. No como se suelen realizar estos cambios en la universidad española, por si alguno quiere hacer algo diferente, de forma voluntaria, sin coordinación, etc." (docente de Ingeniería_ arquitectura) "En 
mi caso, todo mi desarrollo ha sido voluntarista desde el año 2006; la oferta formativa es voluntaria y se ha ofertado durante el último curso. Esta opción de metodología es voluntaria y así, da lugar a disparidad tanto en la oferta como en la valoración de actividad del profesorado, subsistiendo la idea de que a) quien acomete esto es porque le sobra tiempo, b) quien desarrolla este sistema de enseñanza-aprendizaje le dedica menos horas que al sistema tradicional y, por tanto, se penaliza su dedicación docente" (docente de Ingeniería_ arquitectura). "Es necesario un apoyo institucional sin medias tintas. Si el alumnado decide no hacer la parte de trabajo virtual no debe culparse necesariamente al profesorado de ello (siempre que efectivamente no sea culpa suya)" (docente de Ingeniería_ arquitectura). "Escasez de recursos e infraestructura para uso de la metodología" (docente de Ingeniería_ arquitectura). "Falta mucho más interés por parte de las universidades en desarrollar este tipo de metodologías y explicar a los profesores qué se puede hacer y cómo se puede mejorar la docencia con esta metodología y otras" (docente de Ingeniería_ arquitectura). "La ausencia de una estrategia institucional para la transición de la presencialidad a la modalidad b-learning; (b) la falta de apoyo institucional para el desarrollo del b-learning mediante infraestructuras tecnológicas de mayor calidad; (c) la financiación de proyectos de innovación educativa vinculada a equipos docentes y títulos específicos relacionados con el b-learning; (d) la adecuación de la organización institucional a esta modalidad (normativas) y (e) el apoyo decidido a la creación, uso y difusión de Recursos Educativos Abiertos"(docente en Ciencias Sociales y Jurídicas)

\section{Conclusiones}

- Percepción positiva sobre el uso de entornos BL

Hemos podido comprobar en las opiniones de los docentes participantes una valoración positiva sobre las ventajas de utilización de entornos basados en modalidad Blended Learning. Este resultado está en consonancia con otros presentes en la literatura (Vaughan, 2007; Ginns \& Ellis, 2009; Kenney y Newcombe, 2011; Martín-García \& Sánchez-Gómez, 2014; Venkatesh et, al., 2016; Kintu, Zhu, \& Kagambe, 2017, etc.). De igual modo, algunas demandas del profesorado se corresponden con resultados previos en la misma línea (Panda \& Mishra, 2007). Siguiendo el modelo teórico TAM (Technology Acceptance Model), los docentes universitarios perciben positivamente la enseñanza b-learning en el sentido de que les ayudará a conseguir buenos resultados de enseñanza aprendizaje (Utilidad Percibida) aunque su aplicación no esté exenta de dificultades y amenazas: no tienen suficientes conocimientos técnicos en el uso de las TIC (de ahí la importancia de la formación específica previa sobre BL), opinan que falta infraestructura para su implementación (Condiciones Facilitadoras) y creen que mejorará su imagen social y profesional si la utilizan. 
Como hemos comentado en los resultados se constata que existe una actitud favorable hacia la enseñanza $\mathrm{BL}$, más afianzada en los participantes de Ciencias Sociales y Humanidades, que en los de Ciencias. Apenas existen resultados empíricos publicados con los que poder contrastar este resultado a nivel español y escasas referencias a nivel internacional (Teo, et, al. 2015 y Venkatesh, 2016). Desde una perspectiva más institucional, en la mayoría de los estudios se relaciona el contexto de grupo de trabajo en el marco de los distintos ámbitos disciplinares (grupos de innovación docente o de investigación), con el constructo Influencia Social, que explicaría el efecto contagio por medio del liderazgo y prestigio de cierto profesorado sobre sus compañeros en el fomento del uso de nuevas innovaciones docentes como es BL. Más específicamente, se aprecia que casi la mitad de los que manifiestan estar aprendiendo a utilizar metodologías B-L corresponden a docentes del ámbito de las Ciencias Sociales y Humanas, frente a casi un tercio de la rama de "Ciencias". ("actualmente estoy tratando de aprender lo básico sobre la formación b-learning”); la mayor parte de ellos tienen una actitud positiva hacia esta metodología y tienen intención de aplicar b-learning en un breve espacio de tiempo, considerando la formación recibida la principal causa de motivación ("estoy empezando a integrar progresivamente la metodología b-learning en las materias que imparto"). Dentro de este grupo, hay mayor intención en el profesorado de Ciencias frente al de Humanidades o Ciencias Sociales.

Una valoración bastante extendida entre los docentes es que la metodología BL se puede aplicar en cualquier tipo de contexto de formación y piensan que el futuro de los procesos educativos irá en esta línea, como afirman en algunos comentarios:

- Acerca la realidad al alumno y la vincula con la asignatura (Referencia 1); "Facilita el aprendizaje y la comunicación "Es indispensable en la actualidad en la formación superior" (Referencia 13);"Mi idea ha sido siempre combinar las TICs para mejorar el aprendizaje para que fuese significativo y perdurara, y no tanto la memorización de contenidos como la adquisición de competencias, aprendizaje de formas de trabajo útiles en esta área para el futuro (Referencia 20); "Mejora la calidad de la enseñanza, si hay tiempo ayuda al alumno en su interés por la asignatura (Referencia 24)./

\section{- Percepción negativa sobre el uso de entornos BL}

Más de la mitad del profesorado participante opina que la metodología BL presenta dificultades a la hora de implementarla en las instituciones universitarias, principalmente por las actuales condiciones de contratación del profesorado, ausencia de incentivos o reconocimiento, elevado número de estudiantes en las aulas y la falta de tiempo para preparar de esta forma las asignaturas, en línea con otros resultados presentes en la literatura sobre el tema (Samra, Pérez y Castillo, 2010; Del Dujo, Martín-García, Muñoz, 2012; Martín-García, Del Dujo, Muñoz \& Sánchez-Gomez, 2011; etc). Los docentes de ciencias insisten con más frecuencia en el efecto carga de trabajo y en las 
dificultades de control en la ejecución de tareas por parte de los estudiantes, que los de otras ramas de conocimiento:

- "La utilización de B-learning tiene múltiples aspectos positivos para la docencia pero, para el profesor, incrementa enormemente la carga de trabajo ya que son muchas las horas dedicadas a este tipo de docencia fuera del aula. Esta actividad no es reconocida de ninguna manera por parte de las Instituciones. Además, el uso de las TIC en el aula y fuera de ella suele generar bastantes "sobresaltos" debido a problemas técnicos de todo tipo. Se hace necesario mejorar la infraestructura (velocidad en la red, ordenadores no obsoletos, etc.)". (Referencia 27)

- "La motivación del alumnado es muy complicada. Para los alumnos, en general, la evaluación y calificación son, muchas veces, prioritarios frente al aprendizaje". (Referencia 25)

- "Me gustaría destacar la adicional carga de trabajo que implica para el profesorado, que dadas las limitaciones de recursos materiales y actuales del contexto actual, no parece asumible". (Referencia 30)

- "Muchas veces son los alumnos los que no tienen interés en el uso de este sistema. No se leen el material docente, si las actividades complementarias son voluntarias no participan, sin se les evalúa a través de la plataforma intentan copiar los trabajos, etc. (Referencia 33)

- "No existe control sobre la "fiabilidad" del uso que hace un alumno. Esto implica que hay que responsabilizar al estudiante de su propio aprendizaje" (Referencia 38)

- "Si bien el b-learning facilita la labor docente y la participación de muchos alumnos que por diversos motivos no pueden asistir siempre, es cierto que tiende a un cierto aislamiento. El factor deshumanizador puede considerarse tema a tratar". (Referencia 43)

Una posible explicación de este tipo de opiniones puede estar en sistemas de trabajo más analíticos (basados en resolución de problemas y tareas específicas, etc.) en el ámbito de las ciencias que exigen un mayor control en su autoría, frente a tareas más fácilmente personalizables (trabajos de desarrollo, participación en foros, etc.) habituales en la formación en ciencias sociales y/o humanidades en general.

\section{-Propuesta de mejoras para el uso de BL}

De las propuestas de mejora sugeridas por los participantes para poder implementar de forma óptima la metodología BL en las aulas universitarias se infiere que los desarrollos adecuados de estas metodologías de formación dependen de planes estratégicos y acciones que impliquen cambios sustanciales. Entre las peticiones más frecuentes se encuentran las relacionadas con la conceptualización y formación sobre diseños $\mathrm{BL}$, fomento de estrategias de transición de la presencialidad a la enseñanza BL, infraestructuras tecnológicas de mayor calidad, financiación de proyectos de 
innovación vinculados a equipos docentes y títulos formativos en BL, normativas específicas y apoyo a recursos educativos abiertos, menor número de estudiantes por aula, etc. Sostienen que es necesario un mayor reconocimiento sobre la carga en tiempo y esfuerzo que implica su implantación y, en suma, mejorar la motivación de los docentes (saturados "con tantas reformas de los planes de estudio de los Grados"), además de ampliar la formación específica sobre BL, tanto para docentes como para estudiantes, que permita una mayor implicación y responsabilidad de éstos y, todo ello, en definitiva, la mejora de los procesos de enseñanza y aprendizaje en el contexto de la educación superior.

\section{Agradecimientos}

Este estudio ha sido realizado con el apoyo del Ministerio de Economía y Competitividad de España y el Fondo Europeo de Desarrollo Regional (FEDER). Plan I+D+i, Proyecto Ref. EDU20156721.

\section{Referencias}

Anguera, M. T., Blanco-Villaseñor, A., Losada, J. L., Sánchez-Algarra, P., \& Onwuegbuzie, A. J. (2018). Revisiting the difference between mixed methods and multimethods: Is it all in the name?. Quality \& Quantity, 52(6), 2757-2770.

Becker Adams, S., Cummins, M., Davis, A., Freeman, A., Hall Giesinger, C., \& Ananthanarayanan, V. (2017). NMC Horizon Report: 2017 Higher Education Edition. Austin, Texas: The New Media Consortium.

Creswell, J. W. (2013). Qualitative inquiry and research design: Choosing among five approaches (3rd ed.). Los Angeles, CA: SAGE Publications, IncClark, V. L. P. (2019). Meaningful Integration within Mixed Methods Studies: Identifying Why, What, When and How. Contemporary Educational Psychology.

Del Dujo, A.; Martín-García, A.V \& Muñoz, J.M (2012). Análisis del tiempo en los entornos virtuales de formación. Teoria de la Educacion, 21(2), 19-44.

Samra, A. S., Pérez, T., \& Castillo, E. (2010). B-learning en el aprendizaje de inglés con propósitos académicos, a nivel universitario. Multiciencias, 10, 172-177.

Garrison, D. R., \& Vaughan, N. D. (2007). Blended learning in higher education: Framework, principles, and guidelines. San Francisco, CA: Jossey-Bass. https://doi.org/10.1002/9781118269558.fmatter

Ginns, P. \& Ellis, R.A. (2009). Evaluating the quality of e-learning at the degree level in the student experience of blended learning. British Journal of Educational Technology, 40(4), pp.652-663. https://bit.ly/2Cp4j0S.

Guba, E. G., \& Lincoln, Y. S. (1989). Fourth generation evaluation. Sage.

Hall, G. E. (2014). Evaluando los procesos de cambio. Midiendo el grado de implementación (constructos, métodos e implicaciones). REICE. Revista Iberoamericana sobre Calidad, Eficacia y Cambio en Educación, 12(4). https://revistas.uam.es/index.php/reice/article/view/2839. 
Hall, G. E., \& Hord, S. M. (1987). Change in schools: Facilitating the process. New York: State University of New York Press.

Hernández Sampieri, R., Fernández Collado, C., \& Baptista Lucio, P. (2014). Metodología de la investigación. 6a Ed. México: Editorial Mc Graw Hill.

Kenney, J., \& Newcombe, E. (2011). Adopting a blended learning approach: Challenges, encountered and lessons learned in an action research study. Journal of Asynchronous Learning Networks, 15(1), 45-57. https://bit.ly/2FtgJJ5.

Kintu, M.J., Zhu, C., \& Kagambe, E. (2017). Blended learning effectiveness: the relationship between student characteristics, design features and outcomes. International Journal of Educational Technology in Higher Education, 14, 1-20. https://bit.ly/2VRld1g.

Levitt, H. M., Bamberg, M., Creswell, J. W., Frost, D. M., Josselson, R., \& Suárez-Orozco, C. (2018). Journal article reporting standards for qualitative primary, qualitative meta-analytic, and mixed methods research in psychology: The APA Publications and Communications Board task force report. American Psychologist, 73(1), 26.

Miles, M. B., \& Huberman, A. M. (1994). An expanded sourcebook qualitative data analysis (No. 300.18 M5).

Martín-García, A.V. \& Sánchez-Gómez MC. (2014). Modelo predictivo de la intención de adopción de Blended learning en profesores universitarios. Universitas Psychologica, 13 (2), 601-614.

Panda, S., \& Mishra, S. (2007). E-Learning in a Mega Open University: Faculty attitude, barriers and motivators. Educational Media International, 44(4), 323-338. doi: 10.1080/09523980701680854

Rogers, M. E. (2003). Diffusion of innovations. New York, The Free Press.

Sánchez, J. (2003). Integración Curricular de las TIC: Conceptos y Modelos. Revista Enfoques Educacionales, 5(1).

Sánchez-Gómez M.C. \& Martín-García A.V. (2017). Convergence between quantitative and qualitative methodological orientations: mixed models. In: Costa A., Reis L., Souza F., Moreira A. (eds) Computer Supported Qualitative Research. ISQR 2017. Advances in Intelligent Systems and Computing, vol 621, Springer, Cham (2017).

Šumak, B. \& Šorgo, A. (2016). The acceptance and use of interactive whiteboards among teachers: Differences in UTAUT determinants between pre- and post-adopters. Computers in Human Behavior, Vol. 64, 602-620. https://doi.org/10.1016/j.chb.2016.07.037.

Tashakkori, A., \& Teddlie, C. (Eds. (2010). Sage handbook of mixed methods in social \& behavioral research. Sage.

Teo, T., Fan, X., \& Du, J. (2015). Technology acceptance among pre-service teachers: Does gender matter? Australasian Journal of Educational Technology, 31, 235-251.

Vaughan, N. (2007). Perspectives on Blended Learning in Higher Education. International Journal on ELearning; 2007; 6, 1; 81-94.

Venkatesh, Viswanath; Thong, James Y. L.; \& Xu, Xin (2016). Unified Theory of Acceptance and Use of Technology: A Synthesis and the Road Ahead," Journal of the Association for Information Systems: Vol. 17, Iss. 5, Art. 1. https://bit.ly/2sqgsYn. 


\section{António Víctor Martín García}

Universidad de Salamanca

Facultad de Educación. Departamento de Teoría e Historia de la Educación

Paseo de Canalejas, 169. 37003 Salamanca. España

Email: avmg@usal.es

ORCID: https://orcid.org/0000-0002-0216-2754

\section{María Cruz Sánchez Gómez}

Universidad de Salamanca

Facultad de Educación. Departamento de D.O.E y Métodos de Investigación

educativa

Paseo de Canalejas, 169. 37003 Salamanca. España

Email: mcsago@usal.es

ORCID: https://orcid.org/0000-0003-4726-7143

\section{António Pedro Costa}

Universidade de Aveiro

Ludomedia/webQDA e CIDTFF - Centro de Investigação de Didática e Tecnologia na Formação de Formadores, Departamento de Educação da

Universidade de Aveiro

Campus Universitário de Santiago

3810-193 Aveiro. Portugal

Email: apcosta@ua.pt

ORCID: https://orcid.org/0000-0002-4644-5879

Correspondencia:

António Pedro Costa

CIDTFF - Centro de Investigação de Didática e Tecnologia na Formação de

Formadores

Departamento de Educação da Universidade de Aveiro

Campus Universitário de Santiago

3810-193 Aveiro

Data de submissão: Feveveiro de 2018

Data de avaliação: Maio de 2018

Data de publicação: Julho de 2019 